\title{
Final Report for
} "Numerical Methods and Studies of High-Speed Reactive and Non-Reactive Flows"

Donald W. Schwendeman

Rensselaer Polytechnic Institute

November 20, 2002

U.S. Department of Energy

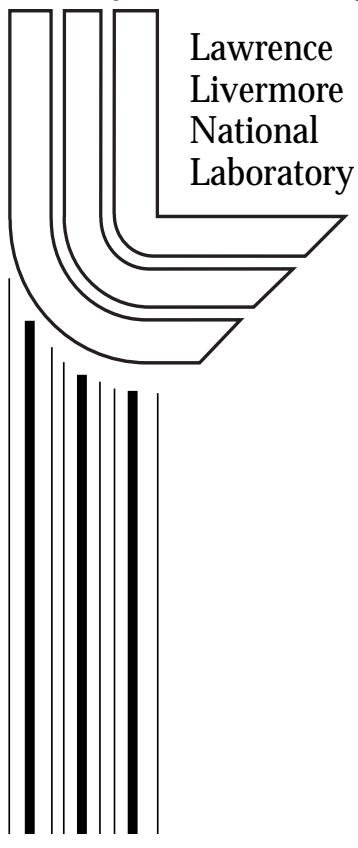




\section{DISCLAIMER}

This document was prepared as an account of work sponsored by an agency of the United States Government. Neither the United States Government nor the University of California nor any of their employees, makes any warranty, express or implied, or assumes any legal liability or responsibility for the accuracy, completeness, or usefulness of any information, apparatus, product, or process disclosed, or represents that its use would not infringe privately owned rights. Reference herein to any specific commercial product, process, or service by trade name, trademark, manufacturer, or otherwise, does not necessarily constitute or imply its endorsement, recommendation, or favoring by the United States Government or the University of California. The views and opinions of authors expressed herein do not necessarily state or reflect those of the United States Government or the University of California, and shall not be used for advertising or product endorsement purposes.

This work was performed under the auspices of the U. S. Department of Energy by the University of California, Lawrence Livermore National Laboratory under Contract No. W-7405-Eng-48.

This report has been reproduced directly from the best available copy.

Available electronically at http://www.doe.gov/bridge

Available for a processing fee to U.S. Department of Energy

and its contractors in paper from

U.S. Department of Energy

Office of Scientific and Technical Information

P.O. Box 62

Oak Ridge, TN 37831-0062

Telephone: (865) 576-8401

Facsimile: (865) 576-5728

E-mail: reports@adonis.osti.gov

Available for the sale to the public from

U.S. Department of Commerce

National Technical Information Service

5285 Port Royal Road

Springfield, VA 22161

Telephone: (800) 553-6847

Facsimile: (703) 605-6900

E-mail: orders@ntis.fedworld.gov

Online ordering: http://www.ntis.gov/ordering.htm

OR

Lawrence Livermore National Laboratory

Technical Information Department's Digital Library

http://www.llnl.gov/tid/Library.html 
Rensselaer Polytechnic Institute

Department of Mathematical Sciences

Troy, New York 12180

\section{Final Report for \\ "Numerical Methods and Studies of High-Speed Reactive and Non-reactive Flow"}

Submitted by:

Donald W. Schwendeman

Associate Professor of Applied Mathematics

\section{FINAL REPORT}

For the period ending September 30, 2002.

Prepared for:

University of California

Lawrence Livermore National Laboratory

Attn: Jill Dunaway

P.O. Box 808, L-561

Livermore, CA 94551

Under:

LLNL subcontract number B523696

RPI contract number A11235 


\title{
Final Report on
}

\author{
LLNL Subcontract B523696 \\ Numerical Methods and Studies of \\ High-Speed Reactive and Non-reactive Flow \\ Donald W. Schwendeman
}

\section{Project Overview}

The work carried out under this subcontract involved the development and use of an adaptive numerical method for the accurate calculation of high-speed reactive flows on overlapping grids. The flow is modeled by the reactive Euler equations with an assumed equation of state and with various reaction rate models. A numerical method has been developed to solve the nonlinear hyperbolic partial differential equations in the model. The method uses an unsplit, shock-capturing scheme, and uses a Godunov-type scheme to compute fluxes and a Runge-Kutta error control scheme to compute the source term modeling the chemical reactions. An adaptive mesh refinement (AMR) scheme has been implemented in order to locally increase grid resolution. The numerical method uses composite overlapping grids to handle complex flow geometries. The code is part of the Overture-OverBlown framework of object-oriented codes $[1,2]$, and the development has occurred in close collaboration with Bill Henshaw and David Brown, and other members of the Overture team within CASC.

During the period of this subcontract, a number of tasks were accomplished, including

- an extension of the numerical method to handle "ignition and grow" reaction models and a JWL equations of state,

- an improvement in the efficiency of the AMR scheme and the error estimator,

- an addition of a scheme of numerical dissipation designed to suppress numerical oscillations/instabilities near expanding detonations and along grid overlaps, and

- an exploration of the evolution to detonation in an annulus and of detonation failure in an expanding channel.

These items are discussed in more detail in Section 3 below.

\section{Dissemination of Results Supported by the Subcontract}

Results of the subcontract work were presented at the following meetings:

- Ninth International Conference on Numerical Combustion, April 7-10, 2002, Sorrento, Italy.

- Fourteenth U.S. National Congress on Theoretical and Applied Mechanics, June 2328, 2002, Blacksburg, Virginia.

- Twelfth International Detonation Symposium, August 11-16, 2002, San Diego, California. 
In addition to presentations given at the meetings above, the following paper is in preparation:

- W.D. Henshaw and D.W. Schwendeman, An adaptive numerical method for high-speed reactive flow on overlapping grids, for submission to J. Comp. Phys..

\section{Project Highlights}

The numerical method has been extended to handle an ignition and growth model of reactive flow [3]. In this model, the explosive mixture consists of two components, a reactant and product. Each component is assigned its own equation of state, typically of JWL form [4], from which is constructed a mixture equation of state under certain closure assumptions such as pressure and temperature equilibrium between the two components. A single variable measures the progress of the reaction. Unlike a simple one-step Arrhenius reaction rate which was implemented in the original code, the ignition and growth model considers a rate function that undergoes sudden changes in form as the progress variable crosses certain values (although the overall rate function remains continuous). The model involves many parameters that require experimental calibration, but our aim is the development of the numerical method to handle the model and the mathematical issues surrounding the model itself. An added difficulty in the implementation of the model is that the assumed equilibrium between states must be maintained throughout the calculation. This is done in a new separate section of code that is designed to handle mixture equations of state. The implementation of this model is new and has yet to be fully exploited to study problems involving detonation propagation in solid explosives.

In a previous subcontract (B515275), an automatic mesh refinement (AMR) scheme was implemented in the code. The scheme is a patch-type refinement scheme similar in flavor to that developed originally by Berger [5]. For a particular domain, a composite overlapping grid is generated using Ogen [6] and this grid becomes the base grid on which AMR grids may be built. As the solution evolves, AMR grids are created, modified or removed based on an error estimate of the solution. The error estimate is based on first and second differences of the solution, as was done in the first implementation of the method, and on a measure of the error due to the source term which has been improved. As mentioned previously, the method handles the source modeling the chemical reactions using a Runge-Kutta errorcontrol scheme. (There are separate chemistry time steps in the method corresponding to an integration of a set of ordinary differential equations.) The error-control scheme estimates the truncation error and this is now used in the AMR error estimator in order to determine whether a grid cell should be tagged for refinement based on the fast scales due to the chemical reaction model.

In addition to improvements in the way the source term contributes to the error estimate for AMR, the AMR grids themselves are now handled more efficiently in the implementation of the method. These improvements involve, in particular, better memory management and more efficient interpolation. This has led to a significant improvement in the computational time spent managing the AMR grids in a typical reactive flow calculation.

A scheme of numerical dissipation is now included in the method. This was done to suppress numerical oscillations/instabilities that have been observed in various reactive flow calculations. A typical case in which the oscillations were observed involves detonation diffraction. If the detonation is expanding along a wall, numerical oscillations in the 
state variables (in the density for example) occur in the direction normal to the wall. For problems of this type, there is very little numerical dissipation provided by the calculation of the numerical fluxes in the Godunov scheme so that an additional scheme of numerical dissipation was considered to be appropriate. A similar problem has been observed near shocks in non-reactive flows [7]. The problem is worse for reactive flows where small numerical oscillation can lead to large errors in the solution due to the state-sensitivity of the solution. Numerical oscillations have also been observed for problems in which a detonation crosses a grid overlap, and our scheme of numerical dissipation helps in these situations as well.

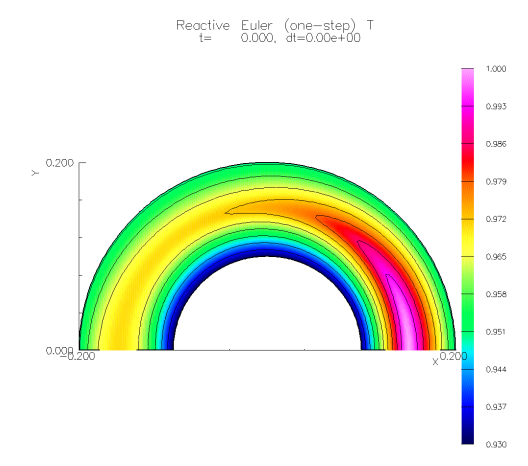

Figure 1: Initial temperature profile.

The numerical method has been used to study a number of problems involving highspeed reactive flow. For example, figure 1 shows an initial temperature profile considered in a study of the evolution to detonation in an annulus. The main goal in the study was to describe mechanisms in which thermal nonuniformities lead to detonation in twodimensional curved geometries, an annulus being a simple example. For this particular case, a one-step Arrhenius reaction model was used and showed the formation of a hot spot which transitioned to detonation as indicated in the sequence of contour plots in figure 2 . These views provide only a small glimpse into the rich variety of mechanism discovered.

Another class of problems for which the numerical method was used concerns detonation diffraction and failure in expanding geometries. When a steady detonation wave encounters an abrupt or smooth expansion, it may fail due to a weakening of the leading shock and a decoupling of the supporting reaction zone behind it. This phenomenon is of basic interest in explosives research and may be studied theoretically by considering suitable mathematical problems involving the reactive Euler equation with various reaction models. We have examined this problem using a three-step, chain-branching reaction model of the type described in [8]. An indication of the behavior is shown in figure 3. In this particular example, an overdriven detonation wave propagates from left to right in the expanding channel. The expansion at the lower wall weakens the leading shock there and lowers its post-shock temperature to a level where the branching step in the three-step reaction model fails. This may be seen in the views of temperature and radical mass fraction in the top row of figure 3. The decrease in the mass fraction of radical behind the leading shock, the key component in the branching step, indicates where the failure occurs. Beyond this point, 

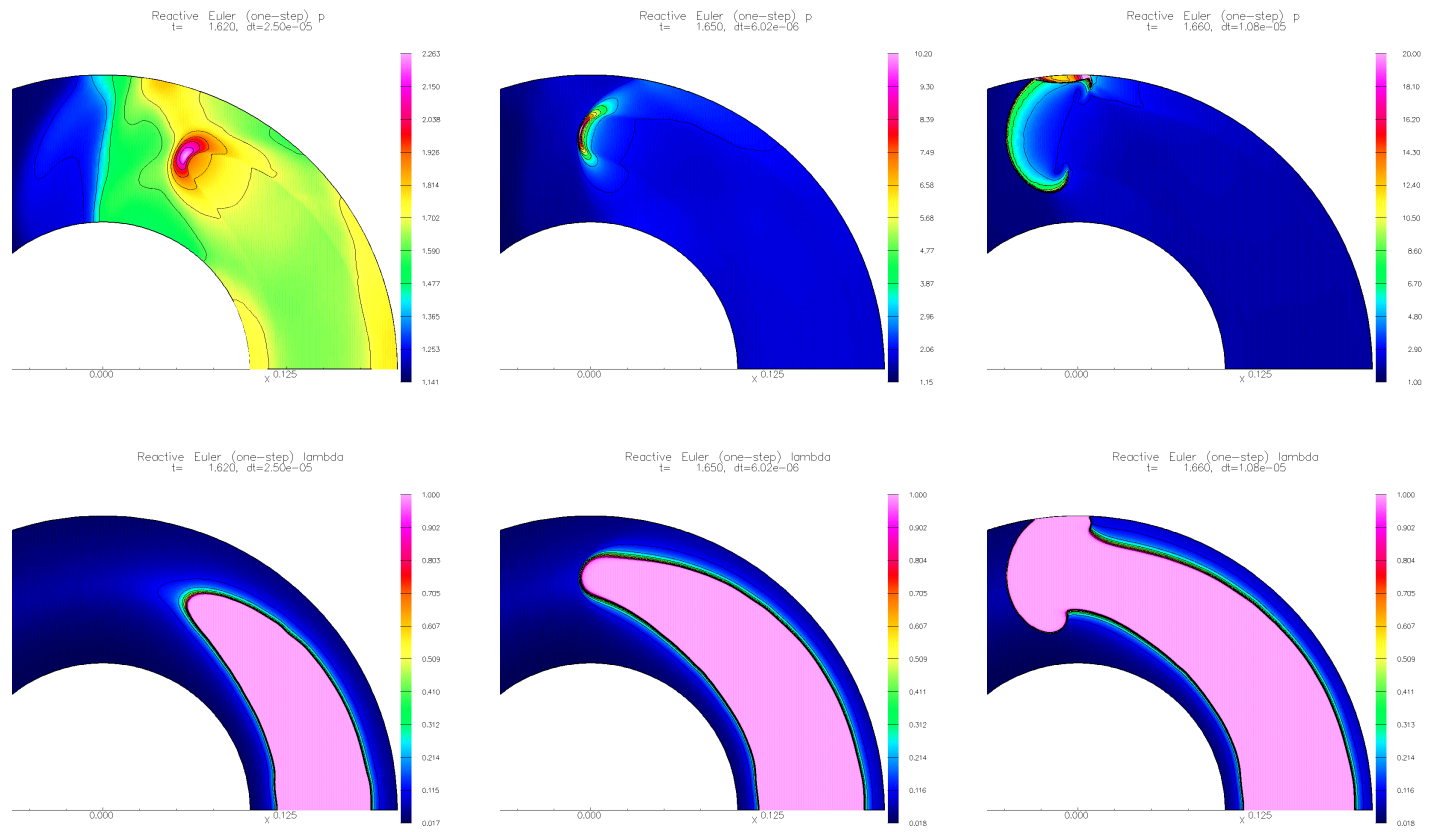

Figure 2: Transition to detonation. Top row is pressure $p$ and bottom row is reaction progress $\lambda$.

there is no significant release in energy behind the shock due to chemical reaction and a region of unreacted explosive is left behind. Of further interest for this channel geometry is a process of re-birth of the detonation due to the strengthening of the shock caused by the Mach reflection in the straight portion of the channel beyond the expansion. This is indicated in the views in the bottom row of figure 3 . Here, the high values of the mass fraction of radical behind the Mach stem indicate the re-birth of the detonation. The views in the last column of the figure show the overlapping grid/AMR grid structure for the calculation. These views are intended to give a flavor of the results and to provide an indication of the use of the numerical method developed.

\section{Concluding Remarks}

Work has continued on the development and use of a numerical method to solve the reactive Euler equations on overlapping grids. A number of improvements have been made to the method and its code during the period of the subcontract. In addition, the code has been used to compute accurate and well-resolved solutions of the equations for a number of problems involving high-speed reactive flow.

\section{References}

[1] W.D. Henshaw, OverBlown: A Fluid Flow Solver For Overlapping Grids, User's Guide, LLNL Research Report UCRL-MA-134288, 1999. 

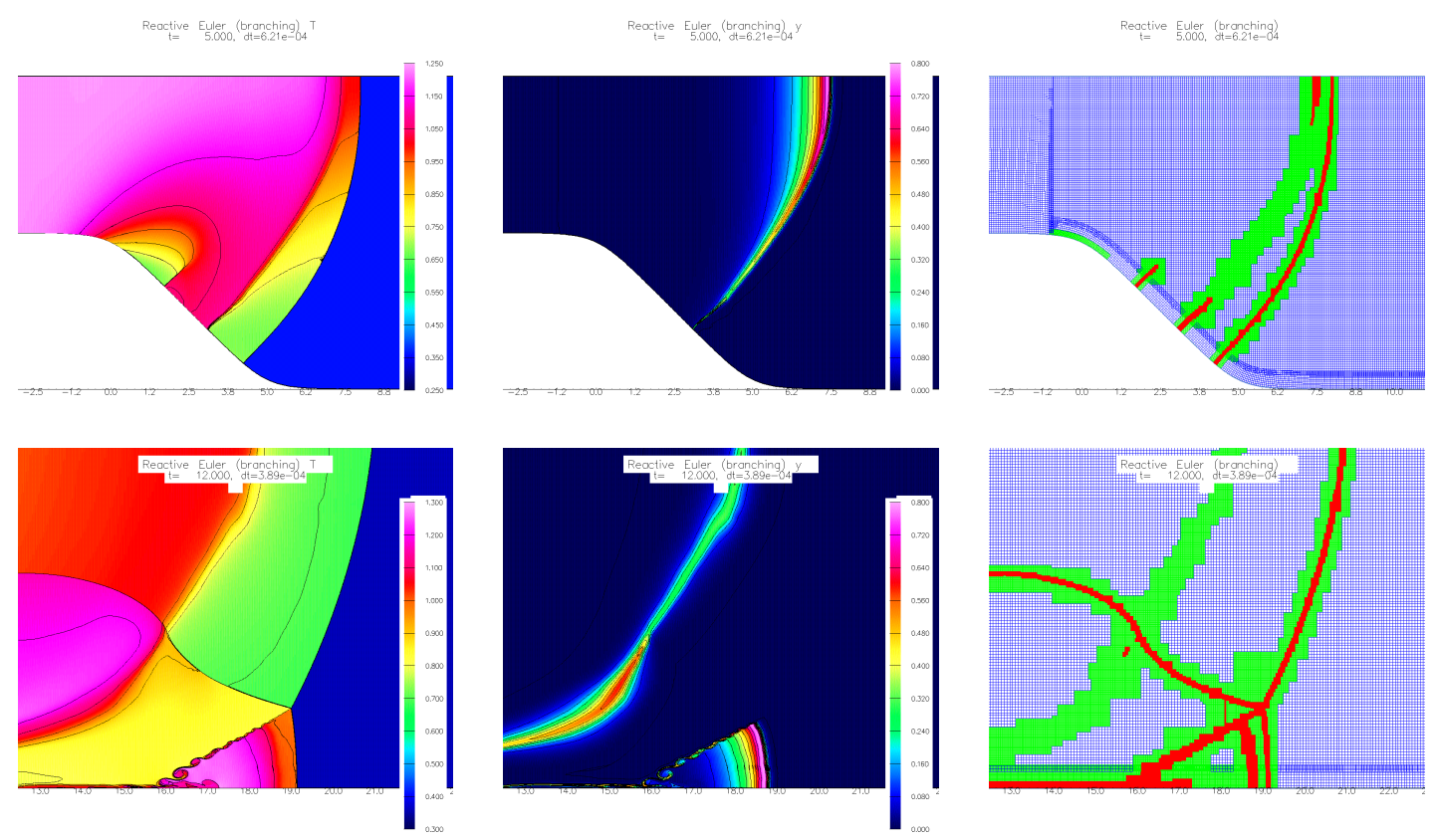

Figure 3: Detonation propagation in an expanding channel. Top row is temperature $T$, radical mass fraction $y$ and the grid for time $t=5$. Bottom row gives the corresponding three views, enlarged near the bottom wall, at $t=12$.

[2] W.D. Henshaw, OverBlown: A Fluid Flow Solver For Overlapping Grids, Reference Guide, LLNL Research Report UCRL-MA-134289, 1999.

[3] C.M. Tarver, J.O. Hallquist and L.M. Erickson, Modeling short pulse duration shock initiation of solid explosives, In 8th Intl. Symp. on Detonation, Albuquerque, NM, 951-961.

[4] L.G. Green, C.M. Tarver and D.J. Erskine, Reaction zone structure in supracompressed detonating explosives, In 9th Intl. Symp. on Detonation, Portland, OR, 670-682.

[5] M.J. Berger And J. Oliger, Adaptive mesh refinement for hyperbolic differential equations, J. Comp. Phys., 53 (1984), 484-512.

[6] W.D. Henshaw, Ogen: An Overlapping Grid Generator for Overture, LLNL Research Report UCRL-MA-132237, 1998.

[7] P. Colella and P. Woodward, The piecewise parabolic method (PPM) for gas dynamical simulations, J. Comp. Phys., (54) 1984, 174-201.

[8] M. ShorT, A.K. KapILA AND J.J. Quirk, The hydrodynamic mechanisms of pulsating detonation-wave instability, Phil. Trans. R. Soc. Lond. A, 357 (1999), 3621-3637. 